\title{
Digitalisierung der Arbeit in der ambulanten Pflege
}

\section{Gestaltung eines sozio-technischen Veränderungsprojekts im Verbundprojekt KoLEGe}

\author{
Peter Bleses, Jens Breuer, Britta Busse, Andreas Friemer, Kristin Jahns, \\ Rebecca Kludig und Stephanie Raudies
}

\subsection{Hintergrund, Zielsetzung und methodisches Vorgehen}

\subsubsection{Hintergrund und Zielsetzung}

Die Digitalisierung der Arbeit macht auch vor der ambulanten Pflege nicht halt. In den Pflegezentralen ist die digital gestützte Arbeitsorganisation und Leistungsabrechnung mittlerweile Standard. Die Digitalisierung dringt zunehmend auch in die Arbeit der Pflegekräfte vor. Dabei handelt es sich vor allem um die Einführung sogenannter

Dieser Beitrag beruht auf den Ergebnissen des vom BMBF und ESF geförderten Verbundprojekts KoLeGe („Interagieren, Koordinieren und Lernen - Chancen und Herausforderungen der Digitalisierung in der ambulanten Pflege“, Laufzeit: 01.09.2016 - 31.12.2019, FKZ: 02L15A01002L15A014).

P. Bleses $(\bowtie) \cdot$ B. Busse $\cdot$ A. Friemer Institut Arbeit und Wirtschaft der Universität und Arbeitnehmerkammer Bremen, Bremen, Deutschland

J. Breuer

Qualitus GmbH, Köln, Deutschland

K. Jahns

Bremer Pflegedienst GmbH, Bremen, Deutschland

R. Kludig

Wirtschafts- und Sozialakademie der Arbeitnehmerkammer Bremen gGmbH,

Bremen, Deutschland

S. Raudies

Johanniter-Unfall-Hilfe e. V., Landesverband Niedersachen/Bremen,

Fachbereich Forschung \& Entwicklung, Elsfleth, Deutschland 
digitaler Tourenbegleiter (MDA = Mobile Digital Assistant), die Pflegekräfte auf ihren Touren mitführen. Auf den meisten MDA läuft die mobile Variante einer Branchensoftware, die auch in der jeweiligen Pflegezentrale genutzt wird. So können die MDA an die Kernprozesse in der jeweiligen Pflegezentrale angekoppelt werden. Die marktgängigen Branchensoftware-Angebote unterscheiden sich zwar in ihren Funktionalitäten, umfassen im Kern aber alle die Tourenplanung, Leistungsdokumentation, Arbeitszeiterfassung und Basisinformationen zu Patient*innen. Hinzu kommen in manchen Angeboten als Erweiterungen jüngst z. B. die mobile Pflegedokumentation, ein Übergabehandbuch und verbesserte Kommunikationsmöglichkeiten.

Die MDA betreffen zwar zunächst vor allem die Pflegeorganisation sowie die Kommunikation über die Pflegeprozesse und noch weniger die eigentliche Pflegearbeit, also die Arbeit am und mit gepflegten Menschen. Aber auch hier hat der MDA-Einsatz Folgewirkungen (z. B. Nutzung bei Patient*innen, vgl. [10]). Allerdings dringt die Digitalisierung weiter in die Pflegearbeit vor, etwa im Rahmen von ,Tele-Care ' (z. B. Fernkontrolle von Vitalparametern) und in viele weitere digitale Anwendungsszenarien in der ambulanten Pflege. Unterstützt werden diese Trends politisch und rechtlich durch die Förderung der Digitalisierung der Arbeitsorganisation und die überbetriebliche Vernetzung der Pflege (Pflegepersonal-Stärkungsgesetz 2019 - PpSG, Digitale-VersorgungGesetz 2019 - DVG).

Indem die Digitalisierung der ambulanten Pflege verstärkt auch die Pflegekräfte und die Pflegearbeit tangiert, dehnt sie sich nicht nur quantitativ aus, sie zieht auch qualitativ andere Anforderungen nach sich. Denn während die Digitalisierung der Verwaltungsprozesse vorwiegend die Arbeit der Verwaltungs- und Führungskräfte anbetraf, deren Arbeit mit digitalisierten Mitteln inzwischen ,Normalfall` ist, betreffen die aktuellen Entwicklungen die Pflegekräfte als eine Gruppe, die mit digital gestützter Arbeit zuvor noch wenig Berührung hatte. Aber der mutmaßlich geringere Kontakt zu digitalen Arbeitsmitteln ist nicht einmal die entscheidende Variable. Bedeutender ist vielmehr, dass Pflegearbeit als Arbeit am und mit Menschen eine berufskulturelle Prägung kennzeichnet, deren Kern in der persönlichen Interaktionsbeziehung liegt. Das muss nicht zwingend mit Technikablehnung einhergehen, stellt Technikeinsatz aber unter Vorbehalte! Zentral ist dabei die Integrationsfähigkeit von Technik in die Pflegearbeit aus Perspektive der Pflegekräfte: Technik sollte Pflegearbeit unterstützen, darf sie aber nicht stören, ersetzen oder mit Zielen belegen, die mit guter Pflege nicht vereinbar sind. Hierzu kann z. B. eine einseitige Orientierung auf Effizienz zählen, die häufig das Motiv der Technikeinführung bildet. Hinzu dürften Sorgen treten, dass digitale Kompetenzanforderungen neben die eigentlichen berufsfachlichen Anforderungen treten - mit der Konsequenz quantitativer und qualitativer Erweiterungen der Anforderungen: Erstens leiden Pflegekräfte aufgrund des immer dramatischer werdenden Personalmangels sowieso schon unter Arbeitsüberlastung. Digitale Unterstützung der Arbeit kann auf lange Sicht vielleicht helfen, stellt aber zunächst einmal einen qualifikatorischen und zeitlichen Zusatzaufwand dar. Zweitens ist für viele Pflegekräfte ohne Erfahrungen mit digitalen Arbeitsmitteln auch ungewiss, ob sie den Anforderungen des individuell kaum beeinflussbaren digitalen Wandels zukünftig genügen können. All das lässt erst einmal 
nichts Gutes für die Perspektiven der Pflegekräfte auf die Entwicklung ihrer Arbeitsqualität erwarten.

Die Berücksichtigung der Arbeitsqualität von Pflegekräften ist also ein wesentlicher Faktor für die erfolgreiche Bewältigung des digitalen Wandels der Pflegearbeit. Dabei geraten die Fragen in den Fokus, welche generellen Bedarfe Pflegekräfte in der ambulanten Pflege und in anderen Pflegebereichen bezüglich ihrer Arbeit haben, wo sie Nutzen in der digitalen Unterstützung der Pflegearbeit für sich sehen und wie sie sich eine gute Einführung und Praxis der digitalen Geräte vorstellen. Es geht dabei um ein Ausloten der Praxisbedarfe mit einem besonderen Augenmerk auf die Verbesserung der Arbeitsqualität mittels digitaler Unterstützung.

Eine solche Perspektive fokussierte das Verbundprojekt KoLEGe am Beispiel der Einführung von MDA in die ambulante Pflege mit folgenden expliziten Aspekten:

- Arbeitsqualität von Pflegekräften im digitalen Wandel der Pflegearbeit,

- Praxisnutzen digitaler Unterstützung der Pflegearbeit und des arbeitsbezogenen Lernens sowie

- Beteiligung von Pflegekräften an der Einführung der MDA und deren arbeitsorganisatorischer Einbindung.

Diese Aspekte wurden bislang in der Analyse und Gestaltung des digitalen Wandels in der Arbeit der Langzeitpflege eher weniger berücksichtigt. Weder wurde die Arbeitsqualität der Pflegekräfte bei der Einführung digitaler Technik gleichermaßen beachtet, noch wurde die Pflegekraftperspektive und deren Expertise systematisch einbezogen. Eine Beteiligung der Pflegekräfte am technischen Entwicklungsprozess ist allerdings obligatorisch, wenn man die Digitalisierung in der Pflege (ebenso wie in anderen Branchen) als einen sozio-technischen Prozess begreift. Darunter ist nicht nur zu verstehen, dass Technikentwicklung in Arbeitsorganisationen kein rein technik-rationaler Prozess ist, sondern dass dieser sozial eingebettet ist. Aus sozio-technischer Perspektive geht es zudem um eine wechselseitige Optimierung und Abstimmung von Technik und Arbeitsorganisation: Technische Innovationen dienen der Organisationsentwicklung, die wiederum Anlässe für weitere technische Innovationen schafft (vgl. [17, 18])

Dabei sollte sich der Blick auch auf die organisationalen Anwendungsbedingungen der Technik im Hinblick auf ihre Sinnhaftigkeit, Versteh- und Handhabbarkeit (im Sinne des Kohärenzgefühls [1]) richten, um diejenigen, die mit der Technik umgehen müssen, nicht zu überfordern.

Im Folgenden schildern wir die Ergebnisse des KoLEGe-Projekts bezüglich der Ziele

- Pflegekräfte bei der Einführung von MDA (und darauf installierter Software) einzubeziehen und ihre Interessen gleichwertig neben denen der Organisation zu berücksichtigen,

- die Arbeitsqualität durch digitale Unterstützung praxis- und nutzer*innengerecht zu verbessern, 
- bedarfsgerechte Technikentwicklung am Beispiel eines Software-Prototyps (KoLEGeApp) in und für die Praxis zu betreiben sowie

- digital gestützte Lernkonzepte und (beispielhaft) Lerninhalte für praxisintegriertes Lernen mittels Mobilgeräten sowie stationäres Lernen am PC zu entwickeln.

\subsubsection{Methodisches Vorgehen}

Das Projekt KoLeGe verwendete den methodischen Ansatz der praxisorientierten Handlungsforschung ([3]; zum Überblick ausführlicher [5]). Hier wird zum einen ein Dialog zwischen und innerhalb verschiedener Gruppen eines Unternehmens (z. B. Geschäftsführung, Pflegedienstleitung, Verwaltungskräfte, Pflegekräfte) hergestellt, in dem über Ziele, Bedarfe und Ressourcenlagen ein konsensorientierter Austausch entsteht. Zum anderen erfolgt auch ein direkter Austausch zwischen Wissenschaft und den im Projekt beteiligten Praxis- und Entwicklungspartnern.

Für die Analyse, Entwicklung, Erprobung und Evaluation wurden vorwiegend qualitative Instrumente eingesetzt: Steuerungskreise, Workshops, Expert*inneninterviews, Tourenbegleitungen, Bürohospitationen. Alle Beteiligten orientierten sich dabei an methodischen Grundsätzen, die zum Teil im Projekt weiterentwickelt wurden:

- Iteratives Vorgehen: Im Anschluss an eine Analysephase wurden die Entwicklungsvorhaben ,kleingearbeitet', um einzelne Schritte der Technik-, Lernkonzept- und Organisationskonzeptentwicklung schneller umsetzen zu können. Die Praxis sollte nicht so lange auf Ergebnisse warten müssen. Hierzu wurden die Einzelmaßnahmen priorisiert und zeitlich gestaffelt. Die Umsetzung, Evaluation und ggf. Neuentwicklung erfolgten auf jeden Entwicklungsschritt bezogen, im weiteren Vorgehen auf die sich aufbauenden Entwicklungsschritte.

- Beteiligungsorientierung: Organisatorisch und/oder technisch ausgelöste Veränderungen von Arbeitsprozessen können sinnvoll nur von denjenigen beurteilt werden, die sie auch operativ umsetzen müssen (hier: Pflege-, Verwaltungs- und operativ tätige Führungskräfte). Sie sind daher in die Gestaltung einzubeziehen (siehe grundlegend [4, 6]).

- Praxis- und Nutzenorientierung: Technik wird nicht um der Technik Willen eingesetzt, sondern muss sich auf konkrete Praxisbedarfe der Pflege-, Verwaltungs- und Führungskräfte beziehen.

- Nutzer*innenorientierung: Pflegekräfte müssen mit unterschiedlichen Voraussetzungen (Alter, Sprache, Fachlichkeit, Technikerfahrung) mit der gleichen Software gut arbeiten können. Barrierearmut und gezielte Förderung der Kompetenzbildung helfen dabei.

- Ressourcenorientierung: Um Überforderungen zu vermeiden, sind die finanziellen und personellen Kapazitäten des Unternehmens sowie die qualifikatorischen Ressourcen und Arbeitsbelastungen der Beschäftigten zu berücksichtigen. 


\subsection{Forschungsergebnisse}

\subsubsection{Analyseergebnisse}

Die Berufskultur der Pflege als „Ethos fürsorglicher Praxis“ [16] orientiert die Pflegekräfte in ihrem Arbeitsanspruch und ihrem Erleben von Arbeitsqualität darauf, gute Pflege“ im Sinne der pflegefachlichen Ansprüche zu leisten. Dabei wird die Pflegearbeit als Arbeit an und mit Menschen als Kernbereich von „Interaktionsarbeit“ [8] bezeichnet, die vor allem die Arbeit in der Langzeitpflege stark prägt (vgl. [2, 19]). Hierzu gehört ganz wesentlich, dass es sich um die aktive Herstellung einer guten Kooperationsbeziehung zwischen Pflegekraft und gepflegtem Menschen (Kooperationsarbeit) handelt, wozu die Pflegekräfte verschiedene Mittel einsetzen müssen (Emotionsarbeit, Gefühlsarbeit, subjektivierendes Arbeitshandeln).

Vor diesem Hintergrund sind die spezifischen Ausprägungen und Anforderungen der ambulanten Pflegearbeit (vgl. [5], grundlegend [7]) zu sehen:

- Vorherrschende Alleinarbeit im Zuhause räumlich verteilter Patient*innen mit hohen Anforderungen an alleinverantwortliches Handeln

- Hohe Kommunikations- und Informationsanforderungen auf vielen, zum Teil wenig verlässlichen Kommunikationswegen

- Zum Teil schwierige Kommunikationssituationen in den Pflegezentralen z. B. bei der Übergabe (viele Übergaben gleichzeitig, keine persönliche Übergabe außerhalb der Bürozeiten)

- Pflegekräfte für gleiche Patient*innen sehen sich nicht zur Übergabe, daher Übergabe der Pflegeinformationen über ,Bande‘ (Pflegezentrale)

- Häufiges Einspringen für ausfallende Kolleg*innen auf den Pflegetouren

- Bedienung von Technik (PC und Smartphone) seitens der Pflegekräfte zum Teil ohne Vorkenntnisse

- Anforderungsreiches Fortbildungsmanagement aufgrund geringer Zeitbudgets der Organisation und der Pflegekräfte

- Schwierige Lernsituation für die Pflegekräfte in Fortbildungen (60-90 min) in der Pflegezentrale, meist am Mittag vor oder nach einer Pflegetour

- Hohe Diversität durch unterschiedliche Qualifikationsgruppen mit unterschiedlichen Einsatzmöglichkeiten, Fachkenntnissen und Kommunikationskompetenzen

Aus diesen Herausforderungen der Arbeit in der ambulanten Pflege sind in gemeinsamen Entwicklungsworkshops drei zentrale Handlungsfelder für die Unterstützung der Pflegekräfte durch MDA abgeleitet worden:

(1) Kommunikationsstrukturen

In der ambulanten Pflege werden diverse Kommunikationskanäle genutzt: 
- Persönliche Übergabe (Face-to-Face) nach dem Frühdienst

- Übergabeformulare

- Übergabe per Telefon

- Pflegedokumentation

- Übergabebuch

- Zettel in Fächer der Pflege- oder Verwaltungskräfte

- Gespräche zwischen Pflegekräften während der Tourenvorbereitung (v. a. beim Frühdienst)

- Tourenplan in der Branchensoftware (wenn bereits ein damit ausgerüstetes MDA mitgeführt wird), in der seitens der Pflegezentrale auch aktuelle Informationen zu Patient*innen vermerkt sein können

Pflegekräfte legen viel Wert auf den persönlichen Austausch mit dem Team in der Pflegezentrale und mit Kolleg*innen über Patient*innen. Sie erkennen jedoch auch mögliche Vorteile in der digital gestützten Kommunikation, v. a.:

- direkt und schnell auf gesicherten Wegen (Datenschutz) mit der Pflegezentrale oder anderen Pflegekräften zu kommunizieren (z. B. per Mitteilungen) sowie

- Übergabeinformationen unterwegs zur Vorbereitung auf Patient*innen möglichst immer verfügbar zu haben (gerade wenn sie bei ihnen bislang unbekannten Patient*innen einspringen müssen).

(2) Informationshilfen für unterwegs

Pflegekräfte würden unterwegs gerne ohne lange Zugriffs- oder Verständniszeiten einige grundlegende Informationshilfen verfügbar haben, z. B.:

- Notfall-Standards (etwa für Verkehrsunfälle) oder Nachbereitung von Notfällen bei Patient*innen,

- ein Lexikon medizinischer Grundbegriffe,

- Standards aus dem betrieblichen Qualitätsmanagement und die Pflegeleistungskomplexe.

(3) Digital gestütztes Lernen

Präsenzveranstaltungen sind für Pflegekräfte auch weiterhin ein wichtiges Weiterbildungsformat, weil Vieles nur im kollegialen Austausch erlernbar ist. Aufgrund ihrer Erfahrungen mit den schwierigen Lernsituationen im Betrieb (v. a. Zeit) stehen sie aber auch dem digital gestützten Lernen positiv gegenüber. E-Learning sollte allerdings nicht zur alleinigen Lernform werden, sondern in einen Methodenmix aus digitalen Lernmodulen und Präsenzphasen in der Lerngruppe eingebunden werden - in ein sogenanntes Blended Learning (vgl. ausführlich [12]). 


\subsubsection{Entwicklungsergebnisse}

\subsubsection{Technik}

Auf Basis der Analysen wurde mit den Pflegekräften (abgestimmt mit Verwaltungs- und Führungskräften) die sog. KoLeGe-App entwickelt (vgl. eingehend [9]). Diese App wurde als beispielhafte nutzerorientierte Anwendung konzipiert und verfolgte zwei übergeordnete Ziele: Erstens sollte sie die analysierten Praxisbedarfe aufnehmen und damit zeigen, wie eine beteiligungsorientierte und an der Verbesserung der Arbeitsqualität ausgerichtete Technikentwicklung gestaltet sein kann. Zweitens sollte sie als Beispiel dafür dienen, wie digitale Technik möglichst so in die Praxis eingeführt werden kann, dass alle Beteiligten (unabhängig von ihren technischen, sprachlichen und kulturellen Qualifikationen und Kompetenzen) sie gut und gerne nutzen können. Die App kann entweder parallel zu einer Branchensoftware oder als alleinige Anwendung genutzt werden. Die Software bietet fünf Funktionsbereiche und ist für den Einsatz auf Smartphones und $\mathrm{PC}$ in einem einheitlichen Design gestaltet (siehe Abb. 29.1).

Abb. 29.1 Startbildschirm der KoLeGe-App (Screenshot Projekt KoLeGe)

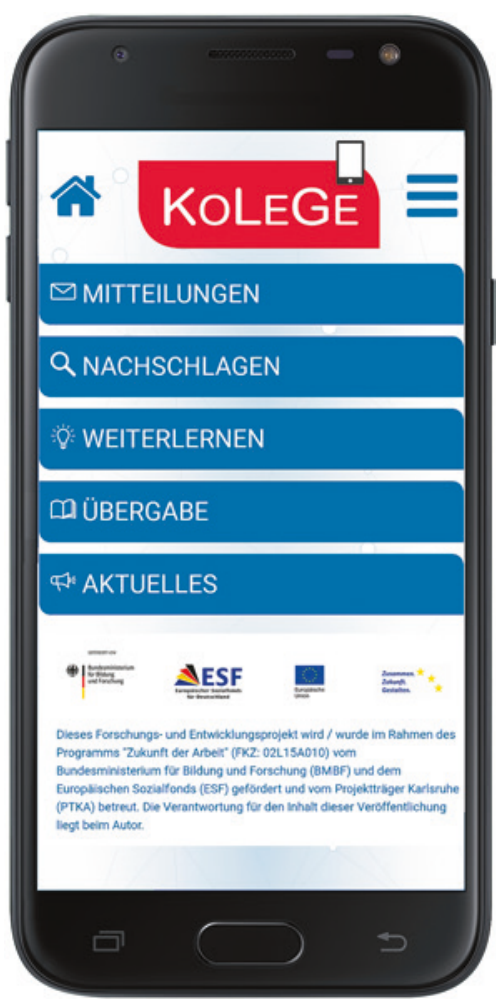


- Die Funktionen Mitteilungen, Übergabe und Aktuelles bilden dabei den Kommunikationsbereich. Mitteilungen besitzen die gleichen Funktionalitäten wie ein E-Mail-System. Es kann nur organisationsintern zwischen den angemeldeten Teilnehmer*innen genutzt werden. Nachrichten werden in einem persönlichen Postfach gespeichert und können nur von den jeweiligen Adressat*innen eingesehen werden. Die Übergabe-Funktion kann - je nach Einrichtung - entweder das analoge ,Übergabebuch', das in der Pflegezentrale liegt, ersetzen. Dann sind die eingetragenen Übergaben von allen berechtigten Personen zu sehen, um unterwegs im Bedarfsfall (z. B. beim Einspringen bei fremden Patient*innen) darauf zugreifen zu können. Oder es ist als ,persönliche Übergabe‘ zwischen einer Pflegekraft und der Pflegezentrale konzipiert. Dann können nur die Führungs- und Verwaltungskräfte und die Pflegekraft darauf zugreifen, die diese Übergabe verfasst hat. Die Funktion Aktuelles ermöglicht es Führungskräften, Informationen automatisiert an alle Pflegekräfte zu senden (z. B. dienstliche Anweisungen).

- Die Funktionen Weiterlernen und Nachschlagen dienen dem Wissensaufbau und als Wissensbasis mit schneller Zugriffsmöglichkeit für unterwegs. Der Bereich Weiterlernen kann als digital gestützte Wissensplattform für das betriebliche Lernmanagement genutzt werden, der einen zeit- und lernortübergreifenden Zugriff auf tiefengestaffelte, multimediale und damit individuell bearbeitbare Lernmaterialien ermöglicht (vgl. [12, 11]).

\subsubsection{Lernen}

Ein digital gestütztes Lernsystem kann die herausfordernde Lernsituation in der ambulanten Pflege entlasten und das Lernen attraktiver gestalten (vgl. zum Folgenden ausführlicher $[12,11]$. Es realisiert eine ständige Verfügbarkeit des anwendbaren Wissens (z. B. auf mobilen Geräten wie MDA), Interaktionsmöglichkeiten im jeweiligen Lernprozess über digitale Vernetzung sowie eine schnelle Aktualisierbarkeit von Inhalten und Medienformaten (z. B. Audio-Text, Video-Anleitungen) in den Lernmodulen.

Konzepte des Blended Learnings stellen dafür einen hybriden Methodenmix aus traditionellen Formen des Präsenslernens und virtuellen E-Learning-Phasen zur Verfügung. Der didaktische Mehrwert von Blended Learning ist die gleichzeitige Vermittlung von fachlicher und Förderung überfachlicher Kompetenz wie z. B. Medien- und Kommunikationskompetenzen, die wiederum maßgeblich sind für die Bildung von Selbstlernkompetenz als wesentliche Grundlage für die Umsetzung des Anspruchs eines lebenslangen Lernens [11].

Nach Einschätzung von Expert*innen (vgl. [13]: S. 84, [14]: S. 145 f.) ist neben der Bereitstellung von adäquaten technischen und organisatorischen Rahmenbedingungen die Bereitschaft der Beschäftigten zentral, diese Lernform am Arbeitsplatz nutzen zu wollen. Um Akzeptanz zu erreichen, muss das digitale Lernangebot daher die personalen Voraussetzungen der Pflegekräfte adressieren (Lernverhalten, Medienkompetenz etc.), was sich in Bezug auf die heterogenen Lerntypen als besondere Herausforderung darstellt - Lernende sind oft entweder über- oder unterfordert. Um sie, dort abzuholen, wo 
sie stehen', ist es notwendig, im Vorfeld die konkreten Bedarfe in Bezug auf die Lernziele und -inhalte bei den Zielgruppen zu ermitteln.

Die Einbindung des digitalen Lernarrangements in die Fortbildungsstrukturen der ambulanten Pflegedienste orientierte sich im KoLeGe-Projekt an den Handlungsfeldern der potenziellen Lernorte ,Pflegezentrale, Pflegetour (Fahrzeug, Wohnung der Patient*innen) und Privatumgebung der Pflegekräfte' und deren systematischer Verknüpfung.

Gemeinsam mit den Pflegekräften wurden beispielhaft zwei digitale Lernmodule zu den Themen ,Sturz‘ und ,Demenz، mit Bezug zu ihren realen Arbeitsfeldern entwickelt, umgesetzt und erprobt.

Die digitalen Lerninhalte sind modular strukturiert und in unterschiedlichen Formaten (Grundlagenmodule, Lernszenarien, Lernerfolgskontrollen etc.) aufbereitet, um eine hohe Flexibilität und Variabilität für eine bedarfsbezogene (vgl. [15]) Nutzung durch die Pflegekräfte zu gewährleisten. Das didaktische Konzept orientiert sich an den Kategorien von Bereitstellung, Zugänglichkeit und Unterstützung, um die heterogenen Lerngewohnheiten, -erfahrungen und -bedarfe der Pflegekräfte aufzunehmen und die unterschiedlichen kognitiven Charakteristika (Lerndauer, Tiefenstaffelung der Inhalte, Selbststeuerung) möglichst adäquat berücksichtigen zu können.

Integrierte Übungen dienen zur individuellen Reflexion der Lernstände und Abschlusstests zur Dokumentation der Fortbildungsteilnahme.

Die Lernplattform, die über die KoLEGe-Software auf mobilen und stationären Endgeräten an allen Lernorten zugänglich ist, basiert auf einer internetbasierten OpenSource-Lösung und wird auf einem geschützten Web-Server administriert.

\subsubsection{Organisation}

Die Entwicklung und Umsetzung der KoLeGe-App, des Lernkonzepts und der beispielhaft erarbeiteten Lerninhalte wurde im Projekt von Entwicklungsaufgaben begleitet, die auf die organisatorische Einbettung der neuen digitalen Technik abzielten (siehe ausführlicher [20]). Dabei ging es um folgende Herausforderungen:

- Akzeptanz aller Beteiligten: Häufig reagieren Pflegekräfte aus Angst, keine Gelegenheit mehr zu haben, persönlich mit ihren Kolleg*innen zu sprechen, mit Ablehnung gegenüber digital gestützter Kommunikation. Sie möchten explizit nicht, dass alles, was digital kommuniziert werden kann, digital kommuniziert werden sollte. Persönlicher Austausch muss also erhalten bleiben.

- Kontrollängste: In einer Branche, die mit minutengenauen Nachweisen arbeitet, werden beim Einsatz digitaler Arbeitsmittel auch Ängste erweiterter Kontrollmöglichkeiten (z. B. durch Live-Tracking) hervorgerufen.

- Abgrenzungsfragen: Digitale Technik ermöglicht grenzübergreifende Kommunikation. Hier besteht die Gefahr, dass Erwerbsarbeit in das Privatleben hineinregiert, dass gesetzliche Ruhezeiten nicht eingehalten und Arbeitszeiten daheim nicht angerechnet werden. 
- Strukturfragen: Durch das Hinzutreten der digitalen Technik wird die Kommunikationsstruktur ohne organisationale Regelungen noch komplexer und unübersichtlicher als zuvor. Im Sinne sozio-technischer Systemgestaltung geht es erst um Organisierung und dann um Technisierung bzw. um die wechselseitige Optimierung von Organisation und dazu passender Technik (vgl. [17, 20]).

- Kompetenzfragen: Kompetenzen bilden gerade in der Pflege eine zentrale Herausforderung, weil der Umgang mit digitalen Medien derzeit weder zur Ausbildung noch zum Berufsbild von Pflegekräften gehört. Dies betrifft fachliche ebenso wie überfachliche Kompetenzen, die sich in Lern- und Veränderungsbereitschaft sowie einem sicheren und reflektierten Umgang mit digital gestützter Technik zeigen [11].

- Datenschutzfragen (Beschäftigte und Patient*innen): Diese sind nicht allein rechtlicher Natur, sondern sie begegnen vielmehr den Beschäftigten als Anforderungen, mit der sie ohne organisationale Hilfen kaum umgehen können oder die sie nicht einschätzen können.

Diese Herausforderungen lassen sich insbesondere mit formellen Nutzungsregeln bearbeiten, um die organisationale Einbettung technisch gestützten Arbeitens in der ambulanten Pflege transparent zu strukturieren. Diese etablieren insbesondere (neue) Arbeits- und Organisationsroutinen, die die Verbindlichkeit der neuen Strukturen sichern sollen. Sie setzen aber zu ihrer Befolgung Akzeptanz voraus. Aus diesem Grunde sollten sie gemeinsam mit den Beschäftigten entwickelt, erprobt und evaluiert werden. Nutzungsregeln sollten so einfach wie möglich und verständlich gestaltet werden, um nicht zu einer Überforderung zu führen. Inhaltlich bedeutet das:

- Verschlankung und Verlässlichkeit: Es werden nur die obligatorischen Kommunikationswege geregelt, bei deren Nutzung auch tatsächlich alles gesehen und weitergegeben werden kann.

- Klarheit: Die Zuordnung der Kommunikationszwecke und -inhalte zu eindeutigen Wegen führt dazu, dass alle wissen, welche Dinge wo zu finden sind.

- Abstimmung zwischen digital gestützten und analogen Kommunikationswegen: Welcher analoge Kommunikationsweg wird durch digitale Kommunikation ersetzt? Wann ist persönliche Kommunikation (z. B. im Rahmen der persönlichen Übergabe nach dem Frühdienst) erwünscht oder verpflichtend?

- Verbindlichkeit: Nur die vorgeschriebenen Kommunikationswege dürfen genutzt werden, um die Struktur zu stärken und Unsicherheiten zu vermeiden.

- Schnelligkeit und Ortsunabhängigkeit: Alles, was möglichst sofort und egal wo zur Kenntnis genommen oder gegeben werden soll, sollte digital gestützt kommuniziert werden.

- Barrierearmut: Digital gestützte Kommunikationsmittel ermöglichen eine bessere Lesbarkeit als handschriftliche Notizen. Digital gestützte Kommunikation erfordert aber auch Schreibfähigkeiten und basale technische Fertigkeiten, die von der Organisation gefördert werden müssen, bevor sie vorausgesetzt werden können. 
- Datenschutz und Datensicherheit: Digitale betriebliche Kommunikationsplattformen stellen ein geschlossenes System dar, das in Hinblick auf Sicherheit anderen Alternativen (einsehbare Notizen, mitzuhörende Telefonate, ungeschützte Messenger) überlegen sein kann, wenn die Sicherheitsvorschriften beachtet werden. Handlungssicherheit ergibt sich nur durch eindeutige, leicht verständliche und praktizierbare Datenschutzregelungen.

- Vertrauliche Kommunikation: Neben öffentlicher Kommunikation müssen persönliche Kommunikationsmöglichkeiten vorgehalten werden, die privat bleiben und nur für adressierte Personen verfügbar sind.

- Beschäftigtendatenschutz: Unternehmen legen im Rahmen von Selbstverpflichtungen oder Betriebsvereinbarungen fest, wie sie mit Fragen der digitalen Kontrolle von Beschäftigten (z. B. mobile Zeit- und Ortserfassung) umgehen. Dies betrifft die Sammlung, Vorhaltung und Auswertung der entsprechenden Daten. Transparenz schafft hier Vertrauen und Akzeptanz.

\subsection{Fazit und Ausblick}

Digitalisierungsprozesse bedeuten tiefgehende Eingriffe in organisationale Strukturen mit weitreichenden, nicht immer im Vorfeld erkennbaren Folgen. Das gilt gerade in der stationären wie ambulanten Langzeitpflege als Interaktionsarbeit mit besonderer Berufskultur und -ethik. Unserem Eindruck nach bedeutet die Digitalisierung der Arbeit selbst dann, wenn diese - wie bei den MDA - in der Hauptsache den pflegeorganisatorischen Rahmen betrifft und noch nicht die Pflegearbeit selbst in ihrem Kern, für viele Pflegekräfte eine Herausforderung. Diese Herausforderung wird umso größer werden, je weiter die Digitalisierung in den Kernbereich pflegerischer Arbeit am und mit dem Menschen vorstößt und das Pflegeverhältnis und die Pflegehandlungen selbst noch stärker betrifft als bislang.

Unseres Erachtens bildet die Akzeptanz dieser ,Landnahme“ des Digitalen im Pflegerischen eher einen Prozess, der seitens der Organisation - und seitens der Bildungsträger, Berufsverbände, Interessenvertretungen usw. - aktiv gestaltet und begleitet werden muss. Vieles wird über Erfahrungen der Pflegekräfte mit der Art und Weise des Technikeinsatzes und dessen organisatorischer Rahmung vermittelt werden. Fehler und Versäumnisse, die hier gemacht werden, können ggf. lange nachwirken, weil sie auch einen unter anderen Umständen als sinnvoll erachteten Technikeinsatz, verbrannt' hätten. Wie bei der Vertrauensbildung gilt auch hier: Akzeptanz aufzubauen, dauert lange - Akzeptanz zerstören, geht schnell.

Je weiter digitale Technik in die eigentliche Pflegearbeit vordringt, desto mehr gilt:

- Sie muss den Pflegekräften nutzen, sonst wird sie nicht (gut) eingesetzt und die Akzeptanz weiterer Digitalisierungsschritte leidet, 
- sie muss beteiligungsorientiert eingeführt (am besten sogar entwickelt) werden, damit sie zu etwas ,Eigenem“ wird und von den Pflegekräften als Expert*innen ihrer Arbeit anerkannt wird,

- sie muss nachvollziehbar und handhabbar sein, damit sie nicht zusätzlich Arbeit und Unsicherheit erzeugt, und

- sie benötigt einen verlässlichen und vertrauensschaffenden organisatorischen Rahmen.

Es empfiehlt sich sehr, nicht alle von oben als sinnvoll und durchführbar eingeschätzten Digitalisierungsschritte auf einmal umzusetzen, sondern schrittweise vorzugehen. Dieses Vorgehen - so hat das KoLEGe-Projekt gezeigt - gibt den Beschäftigten einen gewissen Spielraum, sich an die neuen Anforderungen zu gewöhnen. Es bedeutet auch, dass die Beschäftigten Vertrauen in die Gestaltung des Veränderungsprojekts gewinnen können, das so gestaltet wurde, um sie nicht zu überfordern.

Im Vorfeld und während der Umsetzung der neuen Technik sollten Schulungen mit unterschiedlichen Schwerpunkten angeboten werden, um die Kompetenzen mit dem Umgang digitaler Arbeitsmittel zu stärken (vgl. [11]). Aber in der Praxis zeigt sich, dass Gelegenheiten zum Ausprobieren und zum kollegialen Austausch über die Technik und vor allem die Software und ihr Einsatz in der Praxis fast noch mehr zur Entwicklung technischen Verständnisses beitragen als jede Schulung.

Insgesamt muss die Einführung von digitaler Technik in der ambulanten Pflege damit rechnen, dass die ,digitalen Kompetenzen' der Pflegekräfte sehr unterschiedlich sind. Das hat Folgen nicht nur für die Einführung (die einen sind mit der Technik überfordert, die anderen sind gelangweilt), sondern auch für die Akzeptanz der Technik im Einsatz: Während die einen kämpfen, weil sie mit der Technik im Pflegealltag zurechtkommen müssen, der für sie ohne Technik auch funktioniert hat, sind die anderen unzufrieden, dass sie mit der Technik nicht all das tun können, was sie gerne damit tun würden. Überwie Unterforderung bedürfen der Moderation und des kollegialen Austauschs, um die eingesetzte Technik für alle akzeptierbar zu machen.

\section{Projektpartner und Aufgaben}

- Bremer Pflegedienst GmbH Entwicklung und Erprobung eines Gestaltungskonzepts bei Ersteinführung eines Tablet-Pools in einem privaten Pflegedienst

- Institut Arbeit und Wirtschaft (iaw) der Universität und Arbeitnehmerkammer Bremen

Entwicklung und Erprobung eines Rahmenkonzepts zur Gestaltung sozialer Innovationen beim Einsatz digitaler Technik in sozialen Dienstleistungen/Verbundkoordination

- Johanniter-Unfall-Hilfe e. V.

Entwicklung und Erprobung eines Gestaltungskonzeptes beim Einsatz persönlicher Smartphones in der freien Wohlfahrtspflege 


\section{- Qualitus GmbH, Köln}

Entwicklung und Erprobung einer Kommunikations-, Informations- und Lernsoftware für die digitale Tourenbegleitung

- Wirtschafts- und Sozialakademie der Arbeitnehmerkammer Bremen gGmbH (wisoak)

Entwicklung und Erprobung arbeitsintegrierter E-Learningkonzepte bei digitaler Tourenbegleitung

\section{Literatur}

1. Antonovsky A (1997) Salutogenese. Zur Entmystifizierung der Gesundheit. Tübingen: dgvtVerlag

2. Becke G, Bleses P (2016) Pflegepolitik ohne Arbeitspolitik? Jahrbuch christliche Sozialwissenschaften 57:105-126

3. Becke G, Senghaas-Knobloch E (2011) Dialogorientierte Praxisforschung in organisatorischen Veränderungsprozessen. In: Meyn C, Peter G, Dechmann U, Georg G, Katenkamp O (Hrsg) Arbeitssituationsanalyse. Band 2: Praxistaugliche Beispiele und Methoden. Springer VS, Wiesbaden, S 383-405

4. Bleses, P. (2013): Die direkte Beteiligung von Beschäftigten als Innovation vor der Innovation. In: Klinke, S., Rohn, H. (Hrsg.): Ressourcenkultur. Vertrauenskulturen und Innovationen für Ressourceneffizienz im Spannungsfeld normativer Orientierung und betrieblicher Praxis. Baden-Baden: Nomos, S. 325-341.

5. Bleses P, Busse B, Friemer A, Kludig R, Schnäpp M, Bidmon-Berezinski J, Breuer J, Philippi L (2018) Zwischenbericht des Verbundprojekts KoLeGe. Ergebnisse der Analysephase. Schriftenreihe Institut Arbeit und Wirtschaft 24, Juni 2018, zweite erweiterte Fassung des Berichts. Universität Bremen. https://kolegeprojekt.uni-bremen.de/wp-content/ uploads/2018/07/2018_05_31_ZBW_KoLeGe_Schriftenreihe_final.pdf (eingesehen: 10. Febr 2020)

6. Bleses P, Friemer A, Busse B (2020) Beteiligungsorientierte Digitalisierung der Pflegearbeit: Das Beispiel „digitaler Tourenbegleiter“. In: Kubek V, Velten S, Eierdanz F, Blaudszun-Lahm A (Hrsg) Digitalisierung in der Pflege zur Unterstützung einer besseren Arbeitsorganisation - Potentiale für höhere Arbeitszufriedenheit? Erfahrungen aus Wissenschaft und Praxis. Springer, Wiesbaden

7. Bleses P, Jahns K (2016) Neugestaltung der Koordination und Interaktion in organisatorischen Handlungskontexten sozialer Dienstleistungen. In: Becke G, Bleses P (Hrsg) Interaktion und Koordination. Das Feld sozialer Dienstleistungen. Springer VS, Wiesbaden, S 53-70

8. Böhle F, Stöger U, Weihrich M (2015) Interaktionsarbeit gestalten: Vorschläge und Perspektiven für humane Dienstleistungsarbeit. edition sigma, Berlin

9. Breuer J, Bleses P, Philippi L (2020) Praxisorientierung und Partizipation - Schlüssel für Technikgestaltung in Veränderungsprojekten. In: Bleses P, Busse B, Friemer A (Hrsg) Veränderungsprojekt Digitalisierung der Arbeit in der Langzeitpflege. Springer, Heidelberg

10. Daxberger S (2018) Neue Technologien in der ambulanten Pflege. Wie Smartphones die Pflegepraxis (mit)gestalten. Mabuse, Frankfurt a.M.

11. Friemer A (2020) Digitale Technik droht? Bedroht? Wirklich nur? Kompetenzentwicklung in Veränderungsprojekten. In: Bleses P, Busse B, Friemer A (Hrsg) Veränderungsprojekt Digitalisierung der Arbeit in der Langzeitpflege. Springer, Heidelberg 
12. Kludig R, Friemer A (2020) Blended Learning in der ambulanten Pflege: Partizipative Gestaltung unter Berücksichtigung der Diversität von Pflegekräften. In: Bleses P, Busse B, Friemer A (Hrsg) Veränderungsprojekt Digitalisierung der Arbeit in der Langzeitpflege. Springer, Heidelberg

13. Michel LP (2006) MMB Institut für Medien- und Kompetenzforschung (Hrsg.): Digitales Lernen. Forschung - Praxis - Märkte. Ein Reader zum E-Learning. Essen, Berlin

14. Poppe H (2006) Strategische Überlegungen und Anwenderhinweise für die berufliche Bildung und Weiterbildung. In: Henning PA, Hoyer H (Hrsg) eLearning in Deutschland, Berlin

15. Reglin T (2003) Instrumente selbstorganisierten Lernens - Was neue Medien leisten können. In: Loebe H, Severing E (Hrsg) eLearning für die betriebliche Praxis (Wirtschaft und Weiterbildung: Bd. 30). Bertelsmann, Bielefeld, S 143-157

16. Senghaas-Knobloch E (2008) Care-Arbeit und das Ethos fürsorglicher Praxis unter neuen Marktbedingungen am Beispiel der Pflegepraxis. Berliner J für Soziologie 18:221-243

17. Sträter O (2019) Wandel der Arbeitsgestaltung durch Digitalisierung, in: Zeitschrift für Arbeitswissenschaft (73): 252-260

18. Ulich E (2001) Arbeitspsychologie, 5. Aufl. Schaeffer-Poeschel, Stuttgart

19. Weihrich M, Dunkel W, Rieder K, Kühnert I, Birken T, Herms I (2015) Interaktive Arbeit in der Altenpflege: zwischen Arbeitswelt und Lebenswelt. In: Dunkel W, Weihrich M (Hrsg) Interaktive Arbeit. Theorie, Praxis und Gestaltung von Dienstleistungsbeziehungen. SpringerVS, Wiesbaden, S 181-217

\section{Transfermaterialien}

20. Bleses P, Busse B, Friemer A (Hrsg) (2020) Veränderungsprojekt Digitalisierung der Arbeit in der Langzeitpflege. Springer. Heidelberg

21. Bleses P, Busse B, Friemer A, Behling U, Kludig R, Breuer J, Philippi L (2019) Digitalisierung personenbezogener sozialer Dienstleistungen. Handlungsleitfäden für die Praxis. Universität Bremen, Institut Arbeit und Wirtschaft (iaw) (https://kolegeprojekt.uni-bremen.de/infothek)

Open Access Dieses Kapitel wird unter der Creative Commons Namensnennung 4.0 International Lizenz (http://creativecommons.org/licenses/by/4.0/deed.de) veröffentlicht, welche die Nutzung, Vervielfältigung, Bearbeitung, Verbreitung und Wiedergabe in jeglichem Medium und Format erlaubt, sofern Sie den/die ursprünglichen Autor(en) und die Quelle ordnungsgemäß nennen, einen Link zur Creative Commons Lizenz beifügen und angeben, ob Änderungen vorgenommen wurden.

Die in diesem Kapitel enthaltenen Bilder und sonstiges Drittmaterial unterliegen ebenfalls der genannten Creative Commons Lizenz, sofern sich aus der Abbildungslegende nichts anderes ergibt. Sofern das betreffende Material nicht unter der genannten Creative Commons Lizenz steht und die betreffende Handlung nicht nach gesetzlichen Vorschriften erlaubt ist, ist für die oben aufgeführten Weiterverwendungen des Materials die Einwilligung des jeweiligen Rechteinhabers einzuholen.

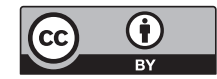

